\title{
Heterogeneous Error-Resilient Scheme for Spectral Analysis in Ultra-Low Power Wearable Electrocardiogram Devices
}

\author{
Soumya Basu' ${ }^{1}$, P. Garcia del Valle ${ }^{1}$, Georgios Karakonstantis ${ }^{2}$, Giovanni Ansaloni ${ }^{3}$ and David Atienza ${ }^{1}$ \\ ${ }^{1}$ Embedded Systems Laboratory, \\ École Polytechnique Fédérale de \\ Lausanne, Switzerland \\ ${ }^{2}$ School of Electronics, Electrical \\ Engineering and Computer Science, \\ Queen's University Belfast, \\ United Kingdom
}

\begin{abstract}
Wearable devices performing advanced bio-signal analysis algorithms are aimed to foster a revolution in healthcare provision of chronic cardiac diseases. In this context, energy efficiency is of paramount importance, as long-term monitoring must be ensured while relying on a tiny power source. Operating at a scaled supply voltage, just above the threshold voltage, effectively helps in saving substantial energy, but it makes circuits, and especially memories, more prone to errors, threatening the correct execution of algorithms. The use of error detection and correction codes may help to protect the entire memory content, however it incurs in large area and energy overheads which may not be compatible with the tight energy budgets of wearable systems.

To cope with this challenge, in this paper we propose to limit the overhead of traditional schemes by selectively detecting and correcting errors only in data highly impacting the end-to-end quality of service of ultra-low power wearable electrocardiogram (ECG) devices. This partition adopts the protection of either significant words or significant bits of each data element, according to the application characteristics (statistical properties of the data in the application buffers), and its impact in determining the output.

The proposed heterogeneous error protection scheme in real ECG signals allows substantial energy savings $(11 \%$ in wearable devices) compared to state-of-the-art approaches, like ECC, in which the whole memory is protected against errors. At the same time, it also results in negligible output quality degradation in the evaluated power spectrum analysis application of ECG signals.
\end{abstract}

Keywords - ultra-low power, embedded systems, wearable health monitors, error tolerance, power spectrum analysis.

\section{INTRODUCTION AND MOTIVATION}

The emergence of wearable devices for long-term acquisition of cardiac signals (electrocardiogram or ECG) promises a paradigm shift in the monitoring of chronic heartrelated conditions. Functionalities of state-of-the-art wearable cardiac sensors are not limited to sensing and (wirelessly) transmitting the acquired data, but they also provide advanced Digital Signal Processing (DSP) capabilities to analyze biosignals on-node and extract clinically-relevant features $[1,2$, $3]$. Diverse applications have been proposed, ranging from the automated detection of epileptic seizures [12] to the predictive risk assessment of atrial fibrillations [13].

In this context, power spectral analysis (PSA) of the heart rate variability (HRV) is among the most widely employed strategies, as it allows the monitoring of various health conditions associated with the heart as well as other organs $[4$, 5], providing valuable frequency-domain medical indicators. The implementation of PSA on ultra-low power embedded devices requires a carefully tailored digital architecture.

A key element in these devices are the memory components, where a significant amount of energy is consumed [1]. To maximize the energy efficiency, an effective approach is to scale down the supply voltage (Vdd). Aggressive supply voltage scaling, leads to quadratic energy savings, but makes circuits (and especially SRAM cells) prone to errors compounding the reliability issues present in nanometer technologies. Larger memory bit-cells [15] and error detection and correction errors (ECC) [17] can help in dealing with errors induced by a scaled Vdd. However, such mechanisms impose large energy and area overheads.

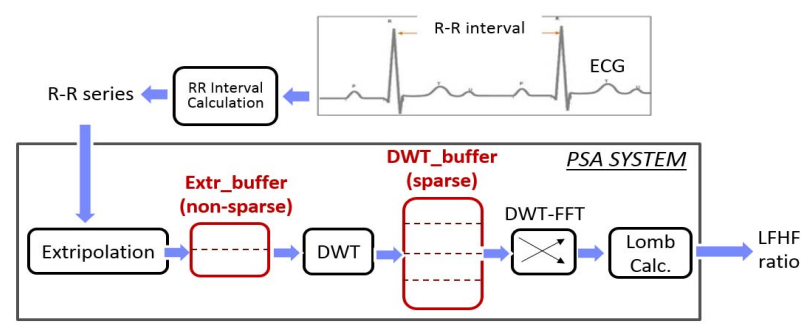

Figure 1: Block Scheme of the PSA Application.

Ensuring the correctness of run-time execution in digital systems is a major challenge, due to the increase in variability derived from technology scaling and near-threshold voltage supplies. A striking alternative, often referred to as approximate computing, is to take advantage of the resilience nature and statistical properties of bio-signal DSP applications such as filtering, features extraction etc. As an energy-saving strategy, the approximate computing paradigm relaxes reliability constraints when errors have a negligible impact from an application perspective. Algorithms in the embedded health monitoring domain operate on noisy acquisitions, while often presenting statistical or qualitative outputs [18]. In such scenarios, in this paper we extend the observations from our previous work [18], and advocate for not needing to provide $100 \%$ exactness in all cases, which is also extremely expensive from an energy efficiency viewpoint. 
In this paper, we propose to investigate the application of the approximate paradigm in bio-signal analysis and feature extraction applications and utilize their statistical properties for limiting the overhead of classical ECC schemes. Our contributions can be briefly described as:

1) We study the statistical properties of a PSA of heart rate variability system and classify data elements in the intermediate steps of the algorithm into significant and lesssignificant based on their contribution to output quality.

2) We apply a significance-based memory protection scheme in PSA systems and evaluate the energy gains and quality compared to traditional full ECC scheme.

3) We exploit the statistical properties of the intermediate elements produced by the PSA system and propose an alternative mitigation scheme. The scheme, rather than trying to correct the errors, only limits their impact on the output quality by replacing the erroneous data with the expected value that is the best available statistical information for the incorrect data.

4) We compare the proposed scheme with existing techniques such as ECC and analyze the energy savings and the quality loss on benchmarks of ECG records in wearable embedded devices.

The rest of this paper is structured as follows: Section II describes the target PSA application in the context of ECG signals. Section III describes the impact of scaled voltages in memories and presents state-of-the-art techniques used to address them. Section III describes the proposed approach for smart wearable sensors. It also analyzes the sparsity of the content of the buffers employed by an optimized PSA implementation, justifying our choice of a hybrid protection scheme. Section IV presents experimental evidence. It also presents experimental evidence showcasing how our proposed scheme can achieve a high quality-of-service in wearable embedded systems, in the presence of errors, by protecting a small fraction of the memory buffers.

\section{POWER Spectral ANAlysis (PSA)}

The power spectral analysis (PSA) of the HRV, has been proposed as a powerful technique to evaluate the autonomic control of the heart rate [10]. Such a system, shown in Figure 1, is composed of 4 essential steps: a) in the first step the time difference between consecutive heartbeats (known as RR intervals) is extracted by processing the recorded ECG within a fixed size window. Due to the non-periodic nature of the RR intervals, Fast Lomb method is considered as the most suitable method for estimating the power spectrum of cardiac signals [6]. According to Fast Lomb, in the next step b) the estimated RR intervals are extrapolated to a fixed size window (i.e. 512 samples), which are then in step c) processed for estimating specific trigonometric functions [6]. A fast method needs to be used at this step for estimating these complex functions, such as the Fast-Fourier-Transform (FFT). In our implementation, we used a Wavelet-based FFT, which was shown recently to be able to take advantage of the sparsity (most elements close to zero) of bio-signals and lead to substantial energy gains [6].

In this modified algorithm initially a Discrete Wavelet Transform (DWT) of size $\mathrm{N}$ is applied on the input signal, followed by butterfly operations similar to the conventional FFT but with modified, simpler coefficients [6] as depicted also in Figure 1. Finally, the Lomb calculator combines the data, estimating the real-time power spectrum information. In the clinical practice, the most used metric derived from PSA is the ratio between the power in low-frequencies (LFP, defined as $0.04-0.15 \mathrm{~Hz}$ ) and high-frequencies (HFP, $0.15-0.4 \mathrm{~Hz}$ ), with $L F H F$ Ratio $=L F P / H F P$. A deviation of the $L F H F$ Ratio above or below normal values is indicative of various health issues [10].

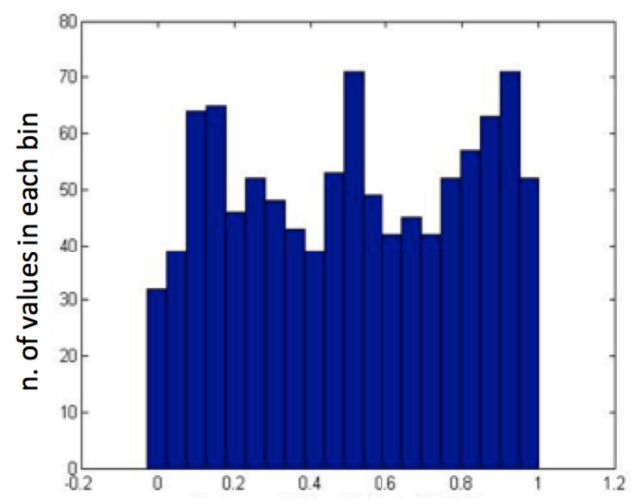

Figure 2: Histogram of Extr_buffer Data (normalized), Distributed in 20 bins. Extr_buffer Presents a Non-Sparse Distribution.

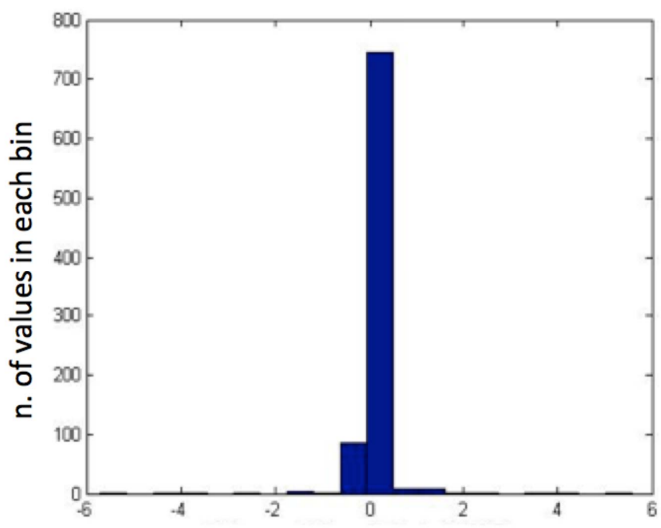

Figure 3: Histogram of DWT_buffer Data (normalized), Distributed in 20 bins. DWT_buffer Presents a Sparse Distribution.

The buffers required by this implementation are the output of the extrapolation step (composed by two sub-arrays) and the output of the DWT transform (composed of four subarrays). 


\section{UNRELIABLE MEMORIES AND PROPOSED APPROACH}

As discussed in Section I, aggressive voltage scaling can induce a non-zero probability of erroneous reads and writes to the memory subsystem. In [7], a bit-flip probability of $0.22 \%$ and $0.07 \%$ are reported for 6-transistors SRAMs supplied at $0.6 \mathrm{~V}$ and $0.65 \mathrm{~V}$ respectively, implemented on a $40 \mathrm{~nm}$ technology. More resilient memory topologies (such as 8transistors and SCMEMs) do allow reliable operations at these voltage levels, however they do incur high area and energy overhead [7]. Alternatively, error detection and correction techniques can be employed to recover from bit-flip events, but they also present non-negligible added complexity, from an area as well as energy perspective.

Our proposed method minimizes such overhead by judiciously employing detection and correction of errors depending on the criticality of the stored data, providing high correctness guarantees only to its most critical part, as dictated by the application characteristics.

Figures 2 and 3 show the typical data distribution across the two buffers used in the PSA application. Figure 3 highlights how the elements of the DWT_buffer are mostly centered on zero, and therefore are sparse, while elements of Extr_buffer (c.f.: Figure 2) have a non-sparse distribution.

Different data distribution patterns require different protection approaches.Intuitively, the most suited protection scheme for the non-sparse Extr_buffer is to protect the Most Significant Bits (MSBs) of every word with an ECC code, as they will have a larger influence on the output. Conversely, a small, but non-zero, probability of a bit-flip in the Least Significant Bits (LSBs) can be allowed (Figure 4).

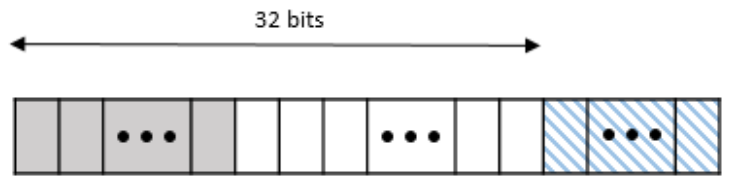

\section{Most Significant Bits that are Protected \\ Unprotected Bits \\ ECC Bits that protect the Most Significant Bits}

Figure 4: A Memory Word in the Extr_buffer

In the case of DWT_buffer, we discriminate between significant and non-significant words, instead of significant bits. In fact, as most of the elements of this buffer are close to zero, it is possible to replace them with their expected value (zero) if an error occurs, which can be detected by employing a simple parity check. For the rest (which, in the PSA application, reside in the low-frequency range) a more expensive error correction capability must be provided; in our case, ECC (Figure 5).

Such partition between significant and non-significant words can be performed statically, i.e. independently from the particular window of inputs being processed. It is in fact derived from the inherent properties of the DWT transform, and the resulting separation of the processed data into high and low frequencies.
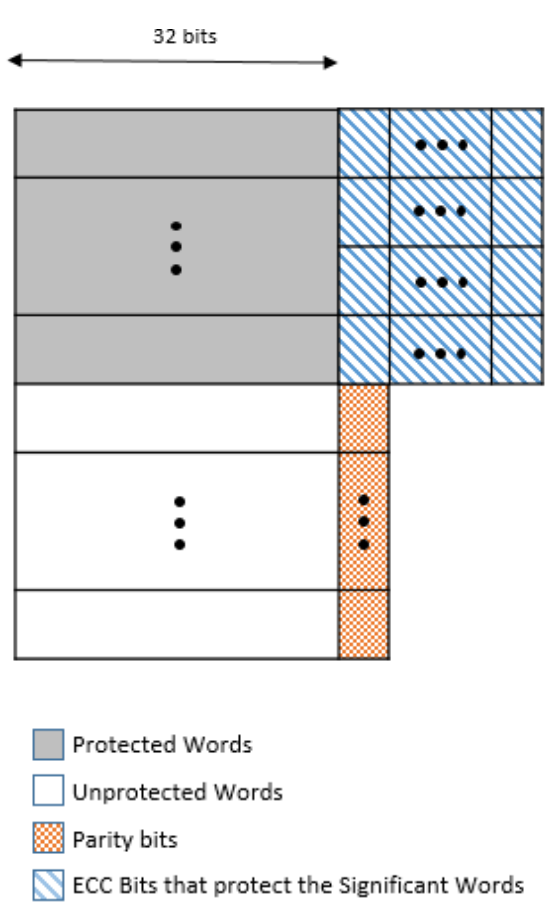

\section{Figure 5: Block Diagram of the DWT_buffer}

It must be noted that a difference exists between the strategies adopted for non-significant bits in non-sparse buffers (e.g.: LSBs in Extr_buffer) and non-significant words in sparse buffers (e.g.: near-zero values in DWT_buffer). In the first case, errors will be completely undetected while, in the second, a parity check will detect the error and invalidate the corresponding word, but no correction will be required. In both cases, the aim is to have a negligible deviation in the end results of the application, while greatly diminishing the protection overhead. In the case of significant bits and words, each bit-flip is detected and corrected, because it highly affects the quality of the output.

\section{EXPERIMENTAL SETUP AND RESULTS}

To evaluate the proposed heterogeneous protection scheme, we developed a high-level fault simulation environment, executing the entire target application. In this way, we evaluated the impact of errors in the intermediate buffers on the quality of the PSA output, which was compared, under different protection schemes, to a fault-free execution. Single bit-flip errors in the buffers are considered with probabilities of $0.07 \%$ and $0.22 \%$, corresponding to the behavior of a 6-transistor SRAM at $0.65 \mathrm{~V}$ and $0.6 \mathrm{~V}$, respectively [6].

Input ECG data was retrieved from the PAF prediction challenge database, available on the Physionet portal [8]. The database includes 100 recordings, of 30 minutes each. We have considered input data windows of 2, 4 and 6 minutes, with an overlap of 1, 3 and 5 minutes, respectively. Results from each recording and each window size are averaged in the presented results. 
To obtain fair results, we employed error masks, forcing bit-flips in random locations of the buffers if they reference to un-protected regions. Different error masks are employed for each processed input window and for each buffer, but the same set is used across all protection configurations. For all buffers, data is represented with 32 bits words. For the Extr_buffer, we explored a protection of the 8, 16, or 32 (all) most significant bits, while for DWT_buffer, we assumed a protection of the $5 \%, 10 \%$ or $15 \%$ of the most significant memory words.

In the following sections, we explored the output degradation induced by bit-flips in the buffers (IV-A), the energy overhead of different protection schemes (IV-B) and the trade-off between energy efficiency and quality of service (IV-C).

\section{A. Error Analysis}

Figures 6 and 7 compare the percentage error in the computation of the LFHF ratio at supply voltages of $0.65 \mathrm{~V}$ (bit-flip probability $=0.07 \%$ ) and $0.6 \mathrm{~V}$ (bit-flip probability $=$ $0.22 \%$ ), respectively.

Results highlight that the selective protection of significant words in sparse buffers can guarantee high-quality results with little overhead. In fact, in the case of a voltage supply of $0.65 \mathrm{~V}$ (Figure 6), less than $1 \%$ error in the LFHF ratio can be achieved by protecting only the most significant $15 \%$ of the significant words in the DWT_buffer, when the non-sparse Extr_buffer is error free. As expected, by reducing the ratio between significant and non-significant words in DWT_buffer, the PSA error increases. Nevertheless, it still remains rather low $(3 \%)$ even when only the $5 \%$ most significant words are protected.

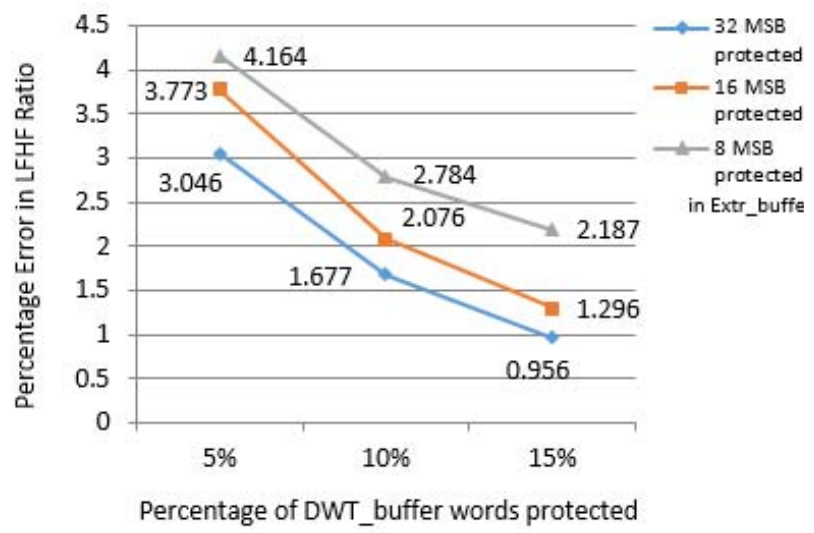

Figure 6: Percentage of Error in the Calculation of the LFP/HFP Ratio under Different Protection Schemes at 0.65V Supply

Conversely, the protection of the non-sparse Extr_buffer presents more challenges; even when protection against bit-flips is provided for the 16 MSBs of each word (which corresponds to protecting half of the buffer content), still a noticeable decrease in quality of service can be noted (square-dotted orange Line in Figure 6).

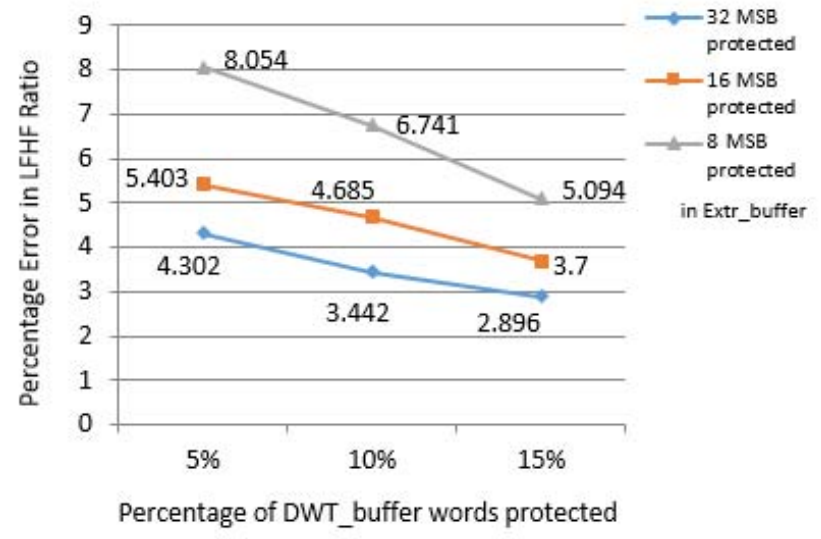

Figure 7: Percentage Error in the Calculation of the LFP/HFP Ratio under Different Protection Schemes at 0.60V Supply

The same trends can be noticed in Figure 7 for a lower voltage supply of $0.6 \mathrm{~V}$, and corresponding higher bit-flip probability. Interestingly, even in this case the deviation in the LFHF ratio, with respect to a fault-free execution, can be bounded to $5 \%$ by allowing errors in the 16 LSBs of Extr_buffer and only checking (but not correcting) errors in $90 \%$ of DWT_buffer.

\section{B. Energy Analysis}

We comparatively evaluated the energy overhead induced by the memory protection configurations by modeling different schemes using CACTI [11] in the McPAT framework [17]. The Extr_buffer requires two buffers, each of $8 \mathrm{~K}$ Bytes, while the DWT_buffer is composed of four buffers of the same size. The operating temperature was assumed to be $300 \mathrm{~K}$. The technology node employed in the simulation of the memories was $40 \mathrm{~nm}$. All wirings were considered to be global, and the interconnect projection was taken as conservative. The memories have a single port used for both reading and writing. Also, for our purpose, we assumed that each of the memories consists of a single bank connected using a bus.

Relevant metrics, output of the CACTI model, consist of the dynamic read and write energies per access and the leakage power of the target memory configurations. To derive the corresponding dynamic energy, we retrieved the number of accesses to the different buffers from the high-level model of the application. In addition, the leakage energy was estimated by considering the execution time of an optimized version of PSA running on an ARM Cortex M4 processor running at $180 \mathrm{MHz}$.

Additional storage is required to support data protection. In the case of Extr_buffer (protection of most significant bits), considering one error detection and correction per memory word, 6 extra ECC bits are required for each word when all 32 data bits are protected. Fewer ECC bits are used when only the most significant part of each word is protected: 5 and 4 bits in the case of 16- and 8- MSB protection, respectively. A maximum of one error occurring in a memory word has been assumed and the number of ECC bits are chosen accordingly as described before. 
In any case, larger number of errors in each word can be also assumed which will require higher order ECC with more redundant bits to detect and correct all the errors. Note that this will lead to higher overheads for the conventional case as opposed to the proposed scheme which acts (for the lesssignificant words) irrespective of the number of errors replacing erroneous words based on the detection of a single error. Therefore, focusing on one failure per word proves the efficacy of the proposed scheme for the popular case of single bit flips while being fair to the conventional schemes.

In the case of DWT buffer (protection of most significant words), a single parity bit is employed for nonsignificant words, allowing error detection. Single error correction is instead supported for significant words by employing a 6-bit error correction code. To simulate this heterogeneous structure in CACTI, we considered the two corner cases where all data is either employing 1-bit parity or 6-bit ECC. To derive the dynamic energy per read access of intermediate configurations, we employed the formula as described in (1).

$$
E_{t=p} * E_{p}+(1-p) * E_{u}
$$

Where $E_{p}$ is the read energy per access in the protected memory, and $E_{u}$ is the read energy per access in the unprotected memory. Also, $p$ is the percentage of considered significant words. $E_{t}$ is the net read energy per access in the heterogeneous memory. The write energy per access and the leakage power of the hybrid memory were also calculated in the same manner.

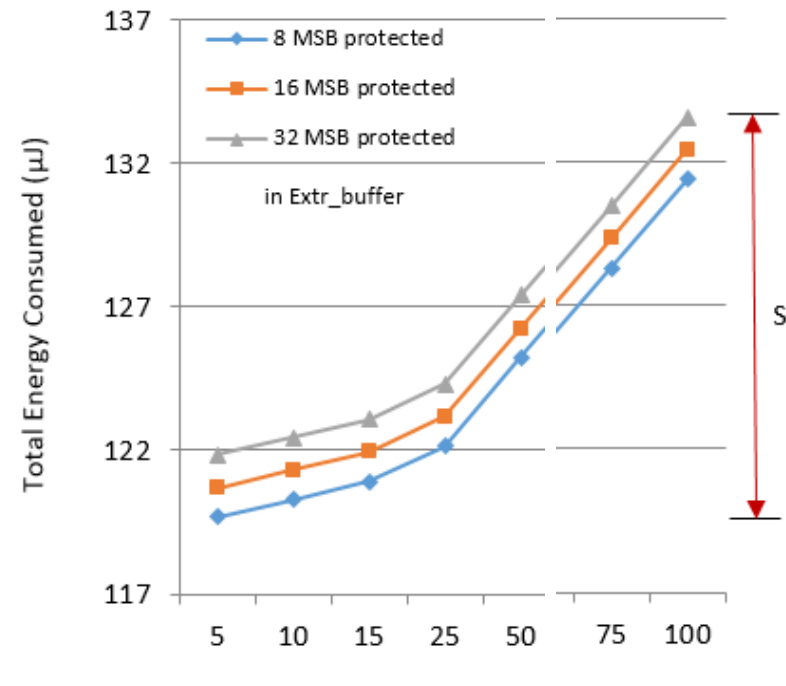

Percentage of DWT_buffer w ords protected

Figure 8: Total Energy Consumption at 0.65V Supply for Different Memory Protection Schi mes for an execution time $\approx 2.67 \mathrm{~s}$

Figure 8 represents a scenario with the maximum possible number of accesses to the hybrid memories for running the application using the RR intervals from a patient recording of 5 minutes. This figure shows the energy consumption of the memory subsystem in an optimized embedded system where power management makes the memory being active only $2.67 \mathrm{~s}$ for the processing of a five minutes recording for a patient. The variable $S$ represents the energy saved with respect to the baseline configuration where $8 \mathrm{MS}$ bits protected in the Extr_buffer and 5\% significant words protected in the DWT_buffer. It shows that full protection of all data entails a significant energy overhead of at least $12 \%$ with respect to baseline configuration. On the other hand, by having a 16-bit MSB protection in Extr_buffer and 10\% of significant words protection in DWT buffer, only a $1.4 \%$ energy overhead is acquired. As reported in Figure 6, this scheme induces a negligible quality-of-service degradation of just $2 \%$ at $0.65 \mathrm{~V}$.

\section{Energy / Quality-of-Service Trade-off}

The proposed methodology can be employed to devise the proper memory configuration when an error constraint is given for the target signal processing application. Alternatively, it can be used to devise the most accurate solution for a fixed energy budget.

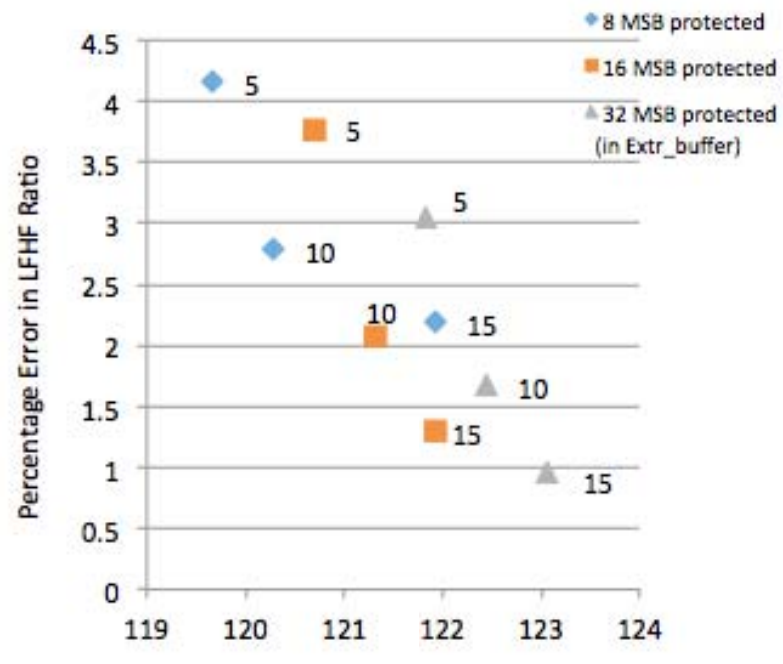

Total Energy Consumed $(\mu)$

The numbers beside the points in the graph signify the percentage of significant words that are protected in the DWT_buffer

Figure 9: Percentage Error in LFHF Ratio and the corresponding energy consumptions under different memory protection schemes at $0.65 \mathrm{~V}$

Figure 9 illustrates this trade-off by plotting the total energy consumed in the buffers for the PSA application against the percentage of error in the computed HFLF error. A percentage of bit-flips of $0.07 \%$ is considered $(\mathrm{Vdd}=0.65 \mathrm{~V})$. For a maximum tolerable error of $2.5 \%$, a solution with 16 MSBs protected in the Extr_buffer and the $10 \%$ most significant data protected in the DWT_buffer is the most energy efficient. Conversely, for an energy budget of $120 \mu \mathrm{J}$, 
the smallest error is achieved by protecting $10 \%$ of the significant data and $8 \mathrm{MSBs}$ in the two buffers.

\section{CONCLUSION}

In this paper, we have explored the energy benefits that can be obtained by applying hybrid data protection schemes to embedded memories for ultra-low power wearable monitoring systems. Working within a well-known, specific application domain allows designers to implement algorithms that exploit significance-based computing to effectively reduce the energy consumption, while keeping the output error under a certain threshold and providing outputs of high enough quality. Our experiments show that by adopting different correctness guaranties in words and bits of varying significance from a digital signal processing viewpoint, the proposed approach can effectively reduce the energy overhead implicit in data protection, while minimally impacting the end-to-end quality of service of the target Power Spectral Analysis application.

The illustrated methodology is applicable in many realworld applications in the embedded health monitoring domain beyond PSA, because they share the same characteristics of processing noisy inputs, providing statistical or qualitative outputs and adopting a sparse representation in intermediate buffers.

Experimental evidence highlights that heterogeneous protection reduces approximately by $11 \%$ the energy budget of the data memory used to store the buffers in real-life wearable ECG analysis systems. Moreover, this new proposed approach tolerates high error rates, potentially allowing more aggressive voltage/frequency scaling at system level. Hence, this observation opens promising venues to be explored in the development of ultra-low power multi-modal wearable embedded systems.

\section{ACKNOWLEDGEMENTS}

This work has been partially supported by the EC FP7 FET SCoRPiO project (grant no. 323872), and ONR-G (grant no. N62909-14-1-N072).

\section{REFERENCES}

[1] Braojos, R., Mamaghanian, H., Junior, A. D., Ansaloni, G., Atienza, D., Rincón, F. J. and Murali, S. "Ultra-Low Power Design of Wearable Cardiac Monitoring Systems". In Proceedings of DAC, June 2014.

[2] Hao, Y. and Foster, R. "Wireless body sensor networks for health-monitoring applications." Physiological measurement, 29(11), R27, 2008.

[3] Massé, F., Bussel, M. V., Serteyn, A., Arends, J. and Penders, J. "Miniaturized wireless ECG monitor for real-time detection of epileptic seizures." ACM Transactions on Embedded Computing Systems (TECS), Vol. 12, no. 4, 102, 2013.

[4] Sörnmo, L., and Laguna, "P. Bioelectrical signal processing in cardiac and neurological applications." Academic Press, 2005.

[5] Chou, C. C., Tseng, S. Y., Chua, E., Lee, Y. C., Fang, W. C. and Huang, H. C., "Advanced ECG processor with HRV analysis for real-time portable health monitoring." In Consumer ElectronicsBerlin (ICCE-Berlin), pp. 172-175. September 2011.

[6] Karakonstantis, G., Sankaranarayanan, A., Sabry, M. M., Atienza, D., and Burg, A., "A quality-scalable and energy-efficient approach for spectral analysis of heart rate variability." In
Proceedings of Design, Automation and Test in Europe Conference and Exhibition (DATE), pp. 1-6, March 2014.

[7] Bortolotti, D., Mamaghanian, H., Bartolini, A., Ashouei, M., Stuijt, J., Atienza Alonso, D., and Benini, L., "Approximate compressed sensing: ultra-low power biosignal processing via aggressive voltage scaling on a hybrid memory multi-core processor." In Proceedings of 2014 IEEE International Symposium on Low Power Electronics and Design (ISLPED 2014).Vol. 1, pp. 40-45.

[8] PhysioBank. Available online: www.physionet.org/physiobank/.

[9] Niskanen, J. P., Tarvainen, M. P., Ranta-Aho, P. O., and Karjalainen, P. A, "Software for advanced HRV analysis." Computer methods and programs in biomedicine, 76(1), pp 7381. 2004.

[10] Akselrod, S., Gordon, D., Ubel, F. A., Shannon, D. C., Berger, A. C., and Cohen, R. J., "Power spectrum analysis of heart rate fluctuation: a quantitative probe of beat-to-beat cardiovascular control." Science, 213(4504), pp 220-222. 1981.

[11] Muralimanohar, Naveen, Rajeev Balasubramonian, and Norman P. Jouppi. "CACTI 6.0: A tool to model large caches." HP Laboratories. 2009.

[12] Massé, Fabien, Martien Van Bussel, Aline Serteyn, Johan Arends, and Julien Penders. "Miniaturized wireless ECG monitor for real-time detection of epileptic seizures." ACM Transactions on Embedded Computing Systems (TECS) 12, no. 4, Article 102 (July 2013), 21 pages.

[13] J. Milosevic, A. Dittrich, A. Ferrante, M. Malek and D. C. Rojas Quirós et al., "Risk Assessment of Atrial Fibrillation: a Failure Prediction Approach", In Proceedings of Computers in Cardiology (CinC), September 2014, Cambridge, MA, pp. 801804.

[14] Zidong Du; Lingamneni, A.; Yunji Chen; Palem, K.; Temam, $\mathrm{O}$.; Chengyong $\mathrm{Wu}$, "Leveraging the error resilience of machinelearning applications for designing highly energy efficient accelerators," In Proceedings of Asia and South Pacific Design Automation Conference (ASP-DAC), January 2014, pp. 201206 ,

[15] Sharma, V.; Cosemans, S.; Ashouei, M.; Huisken, J.; Catthoor, F.; Dehaene, W., "8T SRAM with Mimicked Negative Bit-lines and Charge Limited Sequential sense amplifier for wireless sensor nodes," In Proceedings of ESSCIRC, September 2011, pp. 531-534,

[16] Mohamed M. Sabry, et al.; "OCEAN: An Optimized HW/SW Reliability Mitigation Approach for Scratchpad Memories in Real-Time SoCs". ACM Trans. Embed. Comput. Syst. 13, 4s, Article 138, April 2014, 26 pages.

[17] Sheng Li et al.; "McPAT: An Integrated Power, Area, and Timing Modeling Framework for Multicore and Manycore Architectures". In Proceedings of the 42nd Annual IEEE/ACM International Symposium on Microarchitecture (MICRO 42). ACM, New York, NY, USA, pp. 469-480.

[18] Mohamed Sabry et al.; "Design of energy efficient and dependable health monitoring systems under unreliable nanometer technologies", In Proceedings of the 7th International Conference on Body Area Networks(BodyNets '12). ICST (Institute for Computer Sciences, Social-Informatics and Telecommunications Engineering), ICST, Brussels, Belgium, Belgium, pp. 52-58. 